\title{
De Ritis Ratio (Aspartate Transaminase/Alanine Transaminase) as a Significant Prognostic Factor With Upper Urinary Tract Carcinoma Who Underwent Radical Nephroureterectomy and Adjuvant Chemotherapy
}

\author{
Jee Hwan Yoon ${ }^{1}$, Chang Wook Jeong ${ }^{1}$, Cheol Kwak ${ }^{1}$, Hyeon Hoe Kim ${ }^{1}$, Ja Hyeon Ku ${ }^{1}$, Ji Hyeong Yu², \\ Hyeong Dong Yuk ${ }^{1}$
}

${ }^{1}$ Department of Urology, Seoul National University Hospital, Seoul, Korea

${ }^{2}$ Department of Urology, Inje University Sanggye Paik Hospital, Seoul, Korea

\begin{abstract}
Purpose: To investigate the correlation between preoperative De Ritis ratio (aspartate transaminase $[\mathrm{AST}]$ /alanine transaminase $[\mathrm{ALT}]$ ) and postoperative clinical outcome in patients with upper urinary tract carcinoma (UTUC) who underwent radical nephroureterectomy (RNU) and adjuvant chemotherapy $(\mathrm{ACH})$.

Materials and Methods: We respectively analyzed the clinical and pathological data of 102 patients who underwent RNU and ACH for UTUC. Patients were divided into 2 groups, according to the optimal value of AST/ALT ratio. The effect of the AST/ALT ratio was analyzed by the Kaplan-Meier method and Cox regression hazard models for patients' cancer-specific survival (CSS) and overall survival (OS).

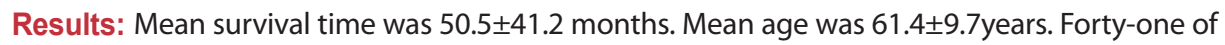
the patients (46.5\%) were in the high AST/ALT group. According to receiver operating characteristic analysis, the optimal AST/ALT ratio was 1.2. In Kaplan-Meier analyses, the high AST/ALT group showed worse outcomes in OS $(p=0.007)$ and CSS $(p=0.011)$. Using Cox regression models of clinical and pathological parameters to predict OS, high AST/ALT ratio (hazard ratio [HR], 5.428; 95\% confidence interval [Cl]; 1.803-16.334; $\mathrm{p}=0.002)$, pathological T3 (pT3) or higher (HR, 1.464; 95\% Cl; 1.156-1.857; $\mathrm{p}=0.002)$, and to predict CSS, high AST/ALT ratio (HR, $4.417 ; 95 \% \mathrm{Cl}$; $1.545-12.632 ; p=0.005)$, and $\mathrm{pT} 3$ or higher $(\mathrm{HR}, 1.475 ; 95 \% \mathrm{Cl} ; 1.172-1.904 ; \mathrm{p}=0.002)$ were determined as independent prognostic factors.

Conclusions: Pretreatment AST/ALT ratio is a significant independent predictor of CSS and OS in advanced UTUC patients receiving systemic ACH after RNU.
\end{abstract}

Key Words: Alanine transaminase, Aspartate transaminase, Chemotherapy, Upper urinary tract carcinoma, Urothelial cell carcinoma
Received October 11, 2021

Revised October 28, 2021

Accepted November 9, 2021

Corresponding author:

Ji Hyeong Yu

Email: uroone@paik.ac.kr

https://orcid.org/0000-0001-8139-0036

Co-corresponding author:

Hyeong Dong Yuk

Email: armenia8@snu.ac.kr

https://orcid.org/0000-0002-5874-9167

\section{INTRODUCTION}

Upper urinary tract carcinoma (UTUC) is a malignant tumor of the urothelium of the urinary tract from calyx to distal ureter. ${ }^{1}$ UTUC is relatively uncommon, and represents only $5 \%-7 \%$ of the tumors that develop in the kidneys. ${ }^{1}$ UTUC accounts for approximately $5 \%-10 \%$ of all genitourinary malignancies. ${ }^{1}$ The disease has a multifocal nature and is diagnosed twice as often in the renal pelvis, as in the ureters. ${ }^{2}$ The standard treatment for localized UTUC is a radical nephroureterectomy 
(RNU), with bladder cuff excision. ${ }^{3}$ RNU series studies performed in the last years have shown that the proportion of locally advanced UTUC is about $60 \%$, the proportion of high-grade tumors is about $70 \%$, and that the proportion of aggressive disease is increasing. ${ }^{4}$ The 5 -year survival rate of UTUC is $20 \%$, and the prognosis is relatively poor. ${ }^{5}$ For these advanced UTUCs, adjuvant chemotherapy $(\mathrm{ACH})$ is recommended to improve longterm prognosis. ${ }^{6}$ However, some advanced UTUC patients progress or the disease recurs to a distant metastasis site. ${ }^{7}$ About $7 \%$ of patients experience metastatic disease. ${ }^{8}$ It is important to accurately predict oncologic outcomes and to properly classify patients at risk for progression or recurrence, to perform additional salvage treatments. For this reason, prognostic factors of survival before treatment are clinically important. We have focused on alanine aminotransferase (ALT) and aspartate aminotransferase (AST), among the many pretreatment predictive factors. ALT and AST are liver enzymes commonly used in different clinical fields, and have been reported in several studies as important biomarkers in various malignant tumors, such as kidney cancer, lung cancer, pancreatic cancer, and colorectal cancer, as well in as chronic liver disease. ${ }^{9-12}$ Recent studies have also shown that UTUC was useful as a preoperative predictor. ${ }^{13-15}$ However, no studies have been reported on the role of AST and ALT as prognostic factors in patients with end-stage UTUC, who received $\mathrm{ACH}$. Therefore, we aimed to identify the prognostic significance of AST and ALT before $\mathrm{ACH}$, in patients with UTUC who underwent RNU and $\mathrm{ACH}$.

\section{MATERIALS AND METHODS}

\section{Ethics Statement}

The study was approved by the Institutional Review
Board of Seoul National University Hospital (IRB No. 2018-11-018). We conducted a retrospective case study and were exempted from obtaining prior consent of the patients. The research protocol was in accordance with the Declaration of Helsinki guidelines.

\section{Study Sample}

We retrospectively analyzed the medical records of 204 patients with advanced UTUC. (pathologic T3 or T4 or pathologic T1- T2N1-N3 without distant metastasis) who were treated in 2 institutions from January 2000 to December 2017. These patients underwent RNU after being diagnosed with UTUC, followed by first line ACH after diagnosis with advanced-stage disease. Of 204 patients, 16 patients suspected of fatty liver and chronic liver disease, and 8 patients suspected of systemic infection and inflammation, were excluded, and 4 patients with incomplete data were also excluded. A total of 176 patients were included in the analyses.

\section{Study Design}

We divided the patients into 2 groups, according to the optimal value of the AST/ALT ratio. The optimal AST/ALT ratio was determined to have the highest sensitivity and specificity in the receiver operating characteristic curve. Patients were divided into 2 groups based on an optimal AST/ALT of 1.2.

ACH usually started within 3 months after surgery, depending on the general condition and renal function of the patients. Laboratory tests including AST and ALT were routinely performed the day before the first cycle of ACH.

The clinical and pathological data collected on patients for this study were: patient age, body mass index (BMI), sex; underlying diseases, such as hypertension and diabetes; RNU surgery methods; pathologic $\mathrm{T}$ stage and $\mathrm{N}$ stage; tumor grade, 
presence of lymphovascular invasion, presence of concurrent carcinoma in situ, presence of ipsilateral hydronephrosis, presence of multifocality, presence of positive surgical margin, regimen and cycle of $\mathrm{ACH}$, American Society of Anesthesiologists (ASA) physical status classification before $\mathrm{ACH}$, and AST and ALT before ACH. Oncologic outcomes data were also collected for mortality, and mortality due to cancer.

Histopathological evaluation of surgical specimens was examined by pathologists skilled in the genital area. The TNM stage and tumor grade were assessed using the 2010 American Joint Committee on Cancer Tumor, Node, Metastases classification, and the World Health Organization/International Society of Urological Pathology consensus classification.

Follow-up was performed every 3 months for the first 2 years, then every 6 months for 2 to 5 years, and every 1 year thereafter. Physical examination, routine laboratory tests, urine analysis, urine cytology, and cystoscopy were performed at every follow-up. Chest computed tomography (CT), abdominal pelvic CT were performed routinely every year.

\section{Statistical Analysis}

Clinical and pathological characteristics were analyzed by independent t-tests between groups. The continuous variables were expressed as mean, standard deviation, median and interquartile range, and the nominal variables were expressed as probability through crossover analysis. The oncologic outcome was analyzed by KaplanMeier survival analysis and log-rank test. Several pretreatment factors affecting the oncologic outcome were analyzed by multivariate Cox proportional hazard regression analysis. A total of 82 patients with high AST/ALT ratios were matched in a 1:1 ratio to 94 patients with low AST/ALT ratios using the nearest neighbor method with 0.02 calibrated. Propensity matching was performed to compensate for the difference between age, sex, BMI, ASA, Tumor location, operative type. The propensity score matching was calibrated and differentiated in most items with a standardized mean difference of less than 0.05 . All statistical tests were performed using IBM SPSS Statistics ver. 22.0 (IBM Co., Armonk, NY, USA), and a p-value of $<0.05$ was considered to indicate statistical significance.

\section{RESULTS}

The clinicopathologic characteristics of all patients are shown in Table 1 . Patients were divided into 2 groups based on an AST/ALT ratio of 1.2. Mean age was 61.4 years and mean AST/ALT ratio was 1.2. Overall mortality was $47.7 \%$, cancer-specific mortality was $44.3 \%$, and mean survival time was 50.5 months. Twenty-eight percent of patients experienced recurrence within the bladder during follow-up. In the high AST/ALT group, the mean age was 64.1 years $(p=0.015)$, the mean mortality rate was $65.9 \%$ and the cancer-specific mortality rate was $61.0 \%(p=0.003$ and $p=0.006)$, higher than the low AST/ALT group. The mean survival time of the high AST/ALT group was 40.4 months $(\mathrm{p}=0.020$ ), which was shorter than the low AST/ALT group. Overall survival (OS) was poor in the high AST/ ALT group compared to the low AST/ALT group $(p=0.007)$, and the cancer-specific survival was also poor in the high AST/ALT group, compared to the low AST/ALT group ( $\mathrm{p}=0.011$ ) (Fig. 1).

In multivariate analysis, age (hazard ratio [HR], 1.041; 95\% confidence interval [CI], 1.003-1.074; $\mathrm{p}=0.039)$, pT3 or higher (HR, 1.464; 95\% CI, 1.156-1.857; $\mathrm{p}=0.002)$, LVI (HR, $1.445 ; 95 \% \mathrm{CI}$, 1.153-1.854; p=0.005), pN1-N2 (HR, 1.342; 95\% CI, 1.011-1.773), pN3 (HR, 3.893; 95\% CI, 2.284-6.648; $\mathrm{p}<0.001$ ), high AST/ALT ratio (HR, 5.428; 95\% CI, 1.803-16.334; $\mathrm{p}=0.002$ ), were significant pre- 
Table 1. Clinicopathological characteristics of patients before and after propensity score matching

\begin{tabular}{|c|c|c|c|c|c|c|}
\hline \multirow{2}{*}{ Variable } & \multicolumn{3}{|c|}{ Before propensity score matching } & \multicolumn{3}{|c|}{ After propensity score matching } \\
\hline & $\begin{array}{l}\text { Low AST/ALT } \\
\quad(\mathrm{N}=94)\end{array}$ & $\begin{array}{l}\text { High AST/ALT } \\
\qquad(\mathrm{N}=82)\end{array}$ & $\mathrm{p}$-value & $\begin{array}{l}\text { Low AST/ALT } \\
\qquad(\mathrm{N}=82)\end{array}$ & $\begin{array}{l}\text { High AST/ALT } \\
\qquad(\mathrm{N}=82)\end{array}$ & $\mathrm{p}$-value \\
\hline Age (yr) & $59.1 \pm 9.9$ & $64.1 \pm 8.9$ & 0.015 & $60.9 \pm 8.7$ & $64.1 \pm 8.9$ & 0.109 \\
\hline $\operatorname{BMI}\left(\mathrm{kg} / \mathrm{m}^{2}\right)$ & $28.8 \pm 8.8$ & $24.2 \pm 9.3$ & 0.020 & $28.3 \pm 7.9$ & $24.2 \pm 9.3$ & 0.037 \\
\hline Male sex & $78(83.0)$ & $54(65.9)$ & 0.109 & $68(82.9)$ & $54(65.9)$ & 0.129 \\
\hline AST & $22.3 \pm 10.0$ & $23.2 \pm 8.5$ & 0.662 & $21.4 \pm 8.8$ & $23.2 \pm 8.5$ & 0.348 \\
\hline ALT & $28.4 \pm 14.2$ & $15.6 \pm 6.8$ & $<0.001$ & $27.2 \pm 13.3$ & $15.6 \pm 6.8$ & $<0.001$ \\
\hline AST/ALT ratio & $0.8 \pm 0.2$ & $1.6 \pm 0.4$ & $<0.001$ & $0.8 \pm 0.2$ & $1.6 \pm 0.4$ & $<0.001$ \\
\hline HTN & $28(29.8)$ & $44(53.7)$ & 0.058 & $24(29.3)$ & $44(53.7)$ & 0.059 \\
\hline $\mathrm{DM}$ & $16(17.0)$ & $16(19.5)$ & 0.623 & $16(19.5)$ & $16(19.5)$ & 0.602 \\
\hline Operation type & & & 0.380 & & & 0.467 \\
\hline Open & $72(76.6)$ & $54(65.9)$ & & $62(75.6)$ & $54(65.9)$ & \\
\hline Laparoscopic & $22(23.4)$ & $28(34.1)$ & & $20(24.4)$ & $28(34.1)$ & \\
\hline ASA PS classification & & & 0.104 & & & 0.061 \\
\hline I & $52(55.3)$ & $26(31.7)$ & & $46(56.1)$ & $26(31.7)$ & \\
\hline II & $42(44.7)$ & $54(65.9)$ & & $36(43.9)$ & $54(65.9)$ & \\
\hline$\geq \mathrm{III}$ & $0(0)$ & $2(2.4)$ & & $0(0)$ & $2(2.4)$ & \\
\hline Pathologic T stage & & & 0.490 & & & 0.648 \\
\hline $\mathrm{pTa}$ & $0(0)$ & $2(2.4)$ & & $0(0)$ & $2(2.4)$ & \\
\hline pT1 & $2(2.1)$ & $6(7.3)$ & & $2(2.4)$ & $6(7.3)$ & \\
\hline pT2 & $10(10.6)$ & $4(4.9)$ & & $6(7.3)$ & $4(4.9)$ & \\
\hline pT3 & $74(78.7)$ & $62(75.6)$ & & $68(82.9)$ & $62(75.6)$ & \\
\hline pT4 & $8(8.5)$ & $8(9.8)$ & & $6(7.3)$ & $8(9.8)$ & \\
\hline Tumor grade & & & 0.802 & & & 0.672 \\
\hline Low & $8(8.5)$ & $4(4.9)$ & & $8(9.8)$ & $4(4.9)$ & \\
\hline High & $86(91.5)$ & $78(95.1)$ & & $74(90.2)$ & $78(95.1)$ & \\
\hline Lymphovascular invasion & $36(38.3)$ & $32(39.0)$ & 0.956 & $32(39.0)$ & $32(39.0)$ & 1.000 \\
\hline Concurrent CIS & $10(10.6)$ & $10(12.2)$ & 1.000 & $8(9.8)$ & $10(12.2)$ & 1.000 \\
\hline Ipsilateral hydronephrosis & $56(59.6)$ & $50(61.0)$ & 0.985 & $50(61.0)$ & $50(61.0)$ & 1.000 \\
\hline Multifocality & $16(17.0)$ & $20(24.4)$ & 0.555 & $16(19.5)$ & $20(24.4)$ & 0.790 \\
\hline Tumor location & & & 0.664 & & & 0.714 \\
\hline Pelvis & $38(40.4)$ & $28(34.1)$ & & $30(36.6)$ & $28(34.1)$ & \\
\hline Ureter & $40(42.6)$ & $34(41.5)$ & & $38(46.3)$ & $34(41.5)$ & \\
\hline Both & $16(17.0)$ & $20(24.4)$ & & $14(17.1)$ & $20(24.4)$ & \\
\hline Pathologic N stage & & & 0.674 & & & 0.609 \\
\hline $\mathrm{pNx}-\mathrm{N} 0$ & $86(91.4)$ & $68(82.9)$ & & $74(90.2)$ & $68(82.9)$ & \\
\hline $\mathrm{pN} 1-\mathrm{N} 2$ & $4(4.3)$ & $8(9.8)$ & & $4(4.9)$ & $8(9.8)$ & \\
\hline $\mathrm{pN} 3$ & $4(4.3)$ & $6(7.3)$ & & $4(4.9)$ & $6(7.3)$ & \\
\hline Positive surgical margin & $8(8.5)$ & $12(14.6)$ & 0.571 & $8(9.8)$ & $12(14.6)$ & 0.736 \\
\hline Neoadjuvant chemotherapy & $2(2.1)$ & $1(1.2)$ & 0.453 & $1(1.0)$ & $1(1.2)$ & 0.521 \\
\hline $\begin{array}{l}\text { Adjuvant chemotherapy } \\
\text { regimens }\end{array}$ & & & 0.430 & & & 0.415 \\
\hline Gemcitabine - carboplatin & $16(17.0)$ & $14(17.1)$ & & $12(14.6)$ & $14(17.1)$ & \\
\hline Gemcitabine - cisplatin & $70(74.4)$ & $62(75.6)$ & & $62(73.2)$ & $62(75.6)$ & \\
\hline G - cisplatin \& & $2(2.1)$ & $6(7.3)$ & & $2(2.4)$ & $6(7.3)$ & \\
\hline G - carboplatin & & & & & & \\
\hline MVAC & $2(2.1)$ & $0(0)$ & & $2(2.4)$ & $0(0)$ & \\
\hline Others & $4(4.3)$ & $0(0)$ & & $4(4.9)$ & $0(0)$ & \\
\hline Chemotherapy cycle & $4.0 \pm 1.6$ & $4.3 \pm 2.3$ & 0.566 & $4.0 \pm 1.6$ & $4.3 \pm 2.3$ & 0.548 \\
\hline Survival duration (mo) & $59.4 \pm 49.2$ & $40.4 \pm 26.7$ & 0.025 & $61.1 \pm 52.1$ & $40.4 \pm 26.7$ & 0.027 \\
\hline Overall mortality & $30(31.9)$ & $54(65.9)$ & 0.003 & $28(34.1)$ & $54(65.9)$ & 0.008 \\
\hline Cancer-specific mortality & $28(29.8)$ & $50(61.0)$ & 0.006 & $26(31.7)$ & $50(61.0)$ & 0.015 \\
\hline
\end{tabular}

Values are presented as mean \pm standard deviation or number (\%).

ALT: alanine aminotransferase, AST: aspartate aminotransferase, BMI: body mass index, HTN: hypertension, DM, diabetes mellitus, ASA PS: American Society of Anesthesiologists physical status, CIS: carcinoma in situ, MVAC: methotrexate, vinblastine, adriamycin and cisplatin. 

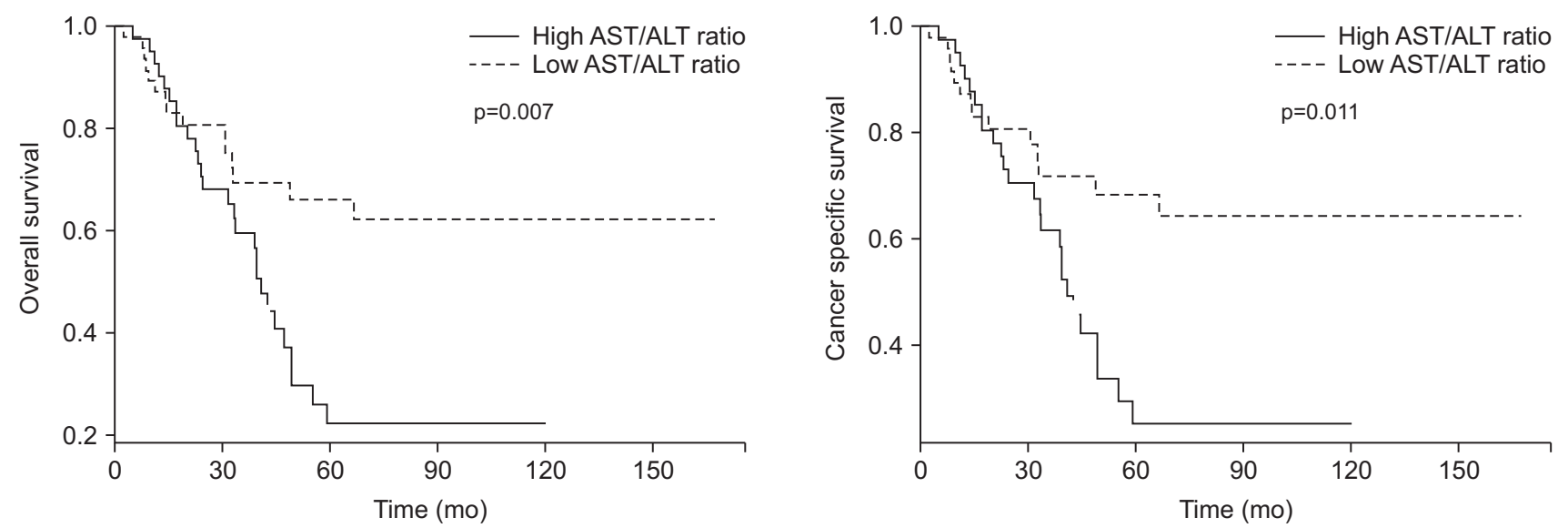

Fig. 1. Overall and cancer-specific survival analyses. Kaplan-Meier survival curves comparing overall and cancer-specific survivals according to the aspartate aminotransferase/alanine aminotransferase (AST/ALT).

Table 2. Multivariate Cox proportional hazards analyses of the AST/ALT ratio on overall survival and cancer-specific survival

\begin{tabular}{|c|c|c|c|c|}
\hline \multirow{2}{*}{ Parameter } & \multicolumn{2}{|c|}{ Overall survival } & \multicolumn{2}{|c|}{ Cancer-specific survival } \\
\hline & $\mathrm{HR}(95 \% \mathrm{Cl})$ & p-value & $\mathrm{HR}(95 \% \mathrm{Cl})$ & $\mathrm{p}$-value \\
\hline Age & $1.041(1.003-1.074)$ & 0.039 & $1.033(0.994-1.075)$ & 0.083 \\
\hline BMI & $0.994(0.932-1.061)$ & 0.842 & $1.011(0.954-1.085)$ & 0.738 \\
\hline \multicolumn{5}{|l|}{ Sex } \\
\hline Men & Reference & & Reference & \\
\hline Women & $0.423(0.114-1.582)$ & 0.198 & $0.482(0.136-1.738)$ & 0.262 \\
\hline \multicolumn{5}{|l|}{ ASA PS classification } \\
\hline I & Reference & & Reference & \\
\hline$\geq \mathrm{II}$ & $1.038(0.790-1.358)$ & 0.207 & $1.048(0.795-1.384)$ & 0.185 \\
\hline \multicolumn{5}{|l|}{ Pathologic T stage } \\
\hline$<\mathrm{pT} 3$ & Reference & & Reference & \\
\hline$\geq \mathrm{pT} 3$ & $1.464(1.156-1.857)$ & 0.002 & $1.497(1.174-1.903)$ & 0.001 \\
\hline \multicolumn{5}{|l|}{ Tumor grade } \\
\hline Low & Reference & & Reference & \\
\hline High & $2.665(0.368-19.883)$ & 0.340 & $2.437(0.323-18.302)$ & 0.386 \\
\hline Lymphovascular invasion & $1.445(1.153-1.854)$ & 0.005 & $1.475(1.172-1.904)$ & 0.002 \\
\hline Concurrent CIS & $0.709(0.151-3.347)$ & 0.658 & $0.877(0.192-4.003)$ & 0.856 \\
\hline Multifocality & $0.662(0.154-2.946)$ & 0.590 & $0.759(0.173-3.194)$ & 0.692 \\
\hline Pathologic N stage & & $<0.001$ & $3.815(2.234-6.497)$ & $<0.001$ \\
\hline $\mathrm{pNx}-\mathrm{N} 0$ & Reference & & Reference & \\
\hline $\mathrm{pN} 1-\mathrm{N} 2$ & $1.342(1.011-1.773)$ & 0.038 & $1.304(0.982-1.735)$ & 0.066 \\
\hline $\mathrm{pN} 3$ & $3.893(2.284-6.648)$ & $<0.001$ & $3.815(2.235-6.493)$ & $<0.001$ \\
\hline Positive surgical margin & $2.155(0.927-5.026)$ & 0.075 & $2.412(1.028-5.674)$ & 0.043 \\
\hline \multicolumn{5}{|l|}{ AST/ALT ratio } \\
\hline Low & Reference & & Reference & \\
\hline High & $5.428(1.803-16.334)$ & 0.002 & $4.417(1.545-12.632)$ & 0.005 \\
\hline
\end{tabular}

AST: aspartate aminotransferase, ALT: alanine aminotransferase, HR: hazard ratio, CI: confidence interval, BMI: body mass index, ASA PS: American Society of Anesthesiologists physical status, CIS: carcinoma in situ.

dictors of poor OS. And pT3 or higher (HR, 1.475; 95\% CI, 1.172-1.904; p=0.002), pN3 (HR, 3.815; 95\% CI, 2.235-6.493; $\mathrm{p}<0.001)$, high AST/ALT ratio (HR, 4.417; 95\% CI, 1.545-12.632; $\mathrm{p}=0.005)$ were significant predictors of poor cancer-specific survival (CSS) (Table 2).

After propensity score matching, the overall mortality rate was significantly higher $(65.9 \%$ 
vs. 34.1\%) in the high AST/ALT group ( $\mathrm{p}=0.008)$ and the cancer-specific mortality rate were also significantly higher (61.0\% vs. $31.7 \%)$ in the high AST/ALT group $(\mathrm{p}=0.015)$ than in the low AST/ ALT group (Table 1). The multivariate Cox analysis showed that a high prechemotherapy AST/ALT ratio was a significant independent predictor of poor prognosis such as OS (HR, 2.095; 95\% CI, 1.090-4.024; $\mathrm{p}=0.026$ ), CSS (HR, 2.074; 95\% CI, 1.053-4.086; $\mathrm{p}=0.035$ ) (Table 3). In addition, pathologic $\mathrm{T}$ stage, $\mathrm{N}$ stage, positive surgical margin are also associated with poor prognosis (Table 3 ).

\section{DISCUSSION}

In this study, we evaluated patients with increased AST/ALT. We observed poor survival outcomes after ACH for advanced UTUC. The AST/ALT ratio was a predictor of OS (HR, 5.428; 95\% CI, 1.803-16.334; $\mathrm{p}=0.002)$ and CSS (HR, 4.417; 95\% CI, 1.545-12.632; $\mathrm{p}=0.005)$. Previous studies on UTUC have also reported that the AST/ALT ratio is a predictor of oncological prognosis. ${ }^{13-15}$ Lee et al. ${ }^{14}$ reported that preoperative high AST/ALT ratio was significantly associated with a poor prognosis of progression-free survival (PFS), CSS, and OS (all p<0.001) in 263 UTUC patient who underwent RNU. They also reported that a high preoperative AST/ALT ratio greater than 1.5 was an independent predictor of PFS (HR, 2.335; 95\% CI, 1.633-3.340; $\mathrm{p}=0.001)$, CSS (HR, 2.550; 1.689-3.851; $\mathrm{p}=0.001)$, and OS (HR, 2.069; 95\% CI, 1.409-3.038; p<0.001) through Cox multivariate analysis. ${ }^{14}$ Cho et al. ${ }^{13}$ reported that a high preoperative AST/ALT ratio

Table 3. Multivariate Cox proportional hazards analyses of the AST/ALT ratio on overall survival and cancer-specific survival after propensity score matching

\begin{tabular}{|c|c|c|c|c|}
\hline \multirow{2}{*}{ Parameter } & \multicolumn{2}{|c|}{ Overall survival } & \multicolumn{2}{|c|}{ Cancer-specific survival } \\
\hline & $\mathrm{HR}(95 \% \mathrm{Cl})$ & p-value & $\mathrm{HR}(95 \% \mathrm{Cl})$ & p-value \\
\hline Age & $1.046(1.005-1.090)$ & 0.029 & $1.031(0.992-1.071)$ & 0.119 \\
\hline BMI & $0.994(0.958-1.031)$ & 0.748 & $1.001(0.964-1.038)$ & 0.983 \\
\hline \multicolumn{5}{|l|}{ Sex } \\
\hline Men & Reference & & Reference & \\
\hline Women & $0.631(0.301-1.323)$ & 0.228 & $0.603(0.276-1.317)$ & 0.205 \\
\hline \multicolumn{5}{|l|}{ ASA PS classification } \\
\hline I & Reference & & Reference & \\
\hline$\geq \mathrm{II}$ & $1.080(0.601-1.939)$ & 0.797 & $1.158(0.630-2.129)$ & 0.636 \\
\hline \multicolumn{5}{|l|}{ Pathologic T stage } \\
\hline$<\mathrm{pT} 3$ & Reference & & Reference & \\
\hline$\geq \mathrm{pT} 3$ & $1.358(1.059-1.744)$ & 0.018 & $1.600(1.251-2.062)$ & 0.002 \\
\hline \multicolumn{5}{|l|}{ Tumor grade } \\
\hline Low & Reference & & Reference & \\
\hline High & $3.321(0.439-25.128)$ & 0.245 & $2.500(0.331-18.907)$ & 0.374 \\
\hline Lymphovascular invasion & $1.366(0.737-2.532)$ & 0.322 & $1.417(0.747-2.687)$ & 0.285 \\
\hline Concurrent CIS & $0.809(0.228-2.271)$ & 0.687 & $0.883(0.313-2.491)$ & 0.814 \\
\hline Multifocality & $0.422(0.178-1.002)$ & 0.051 & $0.628(0.276-1.429)$ & 0.267 \\
\hline Pathologic N stage & & $<0.001$ & & $<0.001$ \\
\hline $\mathrm{pNx}-\mathrm{N} 0$ & Reference & & Reference & \\
\hline $\mathrm{pN} 1-\mathrm{N} 2$ & $1.362(1.051-1.753)$ & 0.018 & $1.702(1.132-2.558)$ & 0.010 \\
\hline $\mathrm{pN} 3$ & $3.301(1.182-9.215)$ & $<0.001$ & $5.364(2.125-13.512)$ & $<0.001$ \\
\hline Positive surgical margin & $2.398(1.018-5.651)$ & 0.046 & $2.882(1.256-6.612)$ & 0.013 \\
\hline \multicolumn{5}{|l|}{ AST/ALT ratio } \\
\hline Low & Reference & & Reference & \\
\hline High & $2.095(1.090-4.024)$ & 0.026 & $2.074(1.053-4.086)$ & 0.035 \\
\hline
\end{tabular}

AST: aspartate aminotransferase, ALT: alanine aminotransferase, HR: hazard ratio, CI: confidence interval, BMI: body mass index, ASA PS: American Society of Anesthesiologists physical status, CIS: carcinoma in situ. 
was significantly associated with a poor prognosis of CSS and OS (all p=0.001), in a multicenter study of 1,049 patients who underwent RNU. They also reported that a preoperative AST/ALT ratio higher than 1.6 was an independent predictor of CSS (HR, 2.49; 95\% CI, 1.70-3.64; $\mathrm{p}=0.001)$ and OS (HR, 1.84; 95\% CI, 1.34-2.52; p=0.001) through Cox multivariate analysis. ${ }^{13}$ In Nishikawa's study of 109 patients with localized UTUC who underwent RNU, a high AST/ALT was significantly independent of extravesical recurrence-free survival (HR, 4.21; 95\% CI, 1.95-9.08; $\mathrm{p}<0.001$ ). In a study of 153 patients with urothelial bladder cancer who underwent radical cystectomy, Gorgel et al. ${ }^{16}$ reported that the preoperative AST/ALT ratio was significantly associated with a poor prognosis of diseasespecific survival (DSS) and OS (all $\mathrm{p}<0.001$ ). They also reported that preoperative AST/ALT ratios higher than 1.3 were independent predictors of DSS (HR, 5.79; 95\% CI, 2.25-15.13; p<0.001) and OS (HR, 2.61; 95\% CI, 1.49-4.56; p<0.001) through Cox multivariate analysis. ${ }^{16}$

The exact mechanism of the relationship between elevated AST/ALT and poor prognosis of cancer patients, including those with UTUC is not yet known. But there are several hypotheses that explain the relationship between AST/ALT ratio and cancer. The most prominent of them is the Warburg effect. ${ }^{17}$ In normal cells, energy is produced by mitochondrial oxidative phosphorylation. However, cancer cells produce energy through lactic acid fermentation glycolysis, rather than oxidative phosphorylation, even under oxygenrich conditions. This aerobic glycolysis is a less efficient pathway than oxidative phosphorylation, and increases the production of metabolites that can contribute to cancer cell proliferation. These metabolites provides anabolic support essential for cancer cell proliferation and growth. ${ }^{17}$ Increased lactate has been suggested to play an important role in maintaining glycolysis, affecting lactate dehydrogenase, the Nicotinamide adenine dinucleotide (NADH)/Nicotinamide adenine dinucleotide (NAD)+ratio, and affecting glucose transport materials. AST plays an important role in the process by relocating $\mathrm{NADH}$ to the mitochondria. ${ }^{18,19}$ In addition, increases in glucose metabolites due to increased lactate, affects the microenvironment of the tumor and promotes local invasion, progression, and metastasis of cancer cells. $^{18,19}$

This study has some limitations. First, since this was a retrospective study of a relatively small number of patients, there may be a recall bias. However, the study population was limited to UTUC patients who underwent RNU and $\mathrm{ACH}$, and that limited the number of patients. Second, we didn't know whether the patient was taking unchecked drugs that would affect AST/ALT.

Despite the limitations, this is the first study to identify the AST/ALT ratio as an important prognostic biomarker of ACH in advanced UTUC. There are many cancer biomarkers, but the AST/ ALT ratio has the merit of cost effectiveness, compared with other methods, and the advantage that it is a quick method that may predict prognosis before cancer treatment.

\section{CONCLUSIONS}

An increased AST/ALT ratio is a significant independent predictor of CSS and OS in advanced UTUC patients receiving systemic ACH after RNU. We suggest that the AST/ALT ratio is a useful and valuable biomarker for predicting oncological outcomes after systemic ACH, as well as RNU in UTUC. The results presented here provide an important insight into the clinical significance of aminotransferase-based markers in postoperative UTUC patients with progressive stage disease, receiving $\mathrm{ACH}$. 


\section{NOTES}

- Conflicts of interest: No potential conflict of interest relevant to this article was reported.

- Funding: This study received no specific grant from any funding agency in the public, commercial, or not-for-profit sectors.

- ORCID

Jee Hwan Yoon: https://orcid.org/0000-0003-4597-5322

Chang Wook Jeong: https://orcid.org/0000-0002-2200-5019

Cheol Kwak: https://orcid.org/0000-0002-1987-2111

Hyeon Hoe Kim: https://orcid.org/0000-0001-6600-4539

Ja Hyeon Ku: https://orcid.org/0000-0002-0391-2342

Ji Hyeong Yu: https://orcid.org/0000-0001-8139-0036

Hyeong Dong Yuk: https://orcid.org/0000-0002-5874-9167

\section{REFERENCES}

1. Siegel RL, Miller KD, Jemal A. Cancer statistics, 2018. CA Cancer J Clin 2018;68:7-30.

2. Favaretto RL, Shariat SF, Chade DC, Godoy G, Adamy A, Kaag M, et al. The effect of tumor location on prognosis in patients treated with radical nephroureterectomy at Memorial Sloan-Kettering Cancer Center. Eur Urol 2010;58:574-80.

3. Rouprêt M, Babjuk M, Compérat E, Zigeuner R, Sylvester RJ, Burger M, et al. European Association of Urology Guidelines on Upper Urinary Tract Urothelial Carcinoma: 2017 update. Eur Urol 2018;73: 111-22.

4. Lughezzani G, Jeldres C, Isbarn H, Sun M, Shariat $\mathrm{SF}$, Widmer $\mathrm{H}$, et al. Temporal stage and grade migration in surgically treated patients with upper tract urothelial carcinoma. BJU Int 2010;105:799804.

5. Ristau BT, Tomaszewski JJ, Ost MC. Upper tract urothelial carcinoma: current treatment and outcomes. Urology 2012;79:749-56.

6. Leow JJ, Martin-Doyle W, Fay AP, Choueiri TK, Chang SL, Bellmunt J. A systematic review and meta-analysis of adjuvant and neoadjuvant chemo- therapy for upper tract urothelial carcinoma. Eur Urol 2014;66:529-41.

7. Cordier J, Sonpavde G, Stief CG, Tilki D. Oncologic outcomes obtained after neoadjuvant and adjuvant chemotherapy for the treatment of urothelial carcinomas of the upper urinary tract: a review. World J Urol 2013;31:77-82.

8. Cosentino M, Palou J, Gaya JM, Breda A, RodriguezFaba O, Villavicencio-Mavrich H. Upper urinary tract urothelial cell carcinoma: location as a predictive factor for concomitant bladder carcinoma. World J Urol 2013;31:141-5.

9. Kiba T, Ito T, Nakashima T, Okikawa Y, Kido M, Kimura A, et al. Bortezomib and dexamethasone for multiple myeloma: higher AST and LDH levels associated with a worse prognosis on overall survival. BMC Cancer 2014;14:462.

10. Lindmark G, Gerdin B, Påhlman L, Bergström R, Glimelius B. Prognostic predictors in colorectal cancer. Dis Colon Rectum 1994;37:1219-27.

11. Rawson NS, Peto J. An overview of prognostic factors in small cell lung cancer. A report from the Subcommittee for the Management of Lung Cancer of the United Kingdom Coordinating Committee on Cancer Research. Br J Cancer 1990;61:597-604.

12. Stocken DD, Hassan AB, Altman DG, Billingham LJ, Bramhall SR, Johnson PJ, et al. Modelling prognostic factors in advanced pancreatic cancer. $\mathrm{Br} \mathrm{J}$ Cancer 2008;99:883-93.

13. Cho YH, Hwang JE, Chung HS, Kim MS, Hwang EC, Jung SI, et al. The De Ritis (aspartate transaminase/alanine transaminase) ratio as a predictor of oncological outcomes in patients after surgery for upper urinary tract urothelial carcinoma. Int Urol Nephrol 2017;49:1383-90.

14. Lee H, Choi YH, Sung HH, Han DH, Jeon HG, Chang Jeong B, et al. De Ritis ratio (AST/ALT) as a significant prognostic factor in patients with upper tract urothelial cancer treated with surgery. Clin Genitourin Cancer 2017;15:e379-85.

15. Nishikawa M, Miyake H, Fujisawa M. De Ritis (as- 
partate transaminase/alanine transaminase) ratio as a significant predictor of recurrence-free survival in patients with upper urinary tract urothelial carcinoma following nephroureterectomy. Urol Oncol 2016;34:417.e9-417.e15.

16. Gorgel SN, Kose O, Koc EM, Ates E, Akin Y, Yilmaz $Y$. The prognostic significance of preoperatively assessed AST/ALT (De Ritis) ratio on survival in patients underwent radical cystectomy. Int Urol Nephrol 2017;49:1577-83.

17. Warburg O. On respiratory impairment in cancer cells. Science 1956;124:269-70.

18. Dorward A, Sweet S, Moorehead R, Singh G. Mitochondrial contributions to cancer cell physiology: redox balance, cell cycle, and drug resistance. J Bioenerg Biomembr 1997;29:385-92.

19. Brizel DM, Schroeder T, Scher RL, Walenta S, Clough RW, Dewhirst MW, et al. Elevated tumor lactate concentrations predict for an increased risk of metastases in head-and-neck cancer. Int J Radiat Oncol Biol Phys 2001;51:349-53. 